\title{
Asset Pricing in a Two-Country Discontinuous General Equilibrium Model
}

\author{
Ciprian Necula \\ DOFIN \\ Academy of Economic Studies \\ Bucharest, Romania \\ email: ciprian.necula@fin.ase.ro
}

First draft: July 52008

\begin{abstract}
The aim of this paper is to develop a framework for asset pricing in a continuous time general equilibrium model for a two country Lucas type economy. The model assumes that the output in the two countries follows a jump-diffusion stochastic process characterized by constant growth rates and volatilities and by log-normal amplitude of the jumps. Using this specification we deduce the fundamental evaluation equations for financial assets as well as a formula for the price of exchange rate options in this economy.
\end{abstract}

Keywords: general equilibrium model, two-country Lucas economy, exchange rate, asset pricing, exchange rate options, jump-diffusion.

JEL Classification: C02, C61, D50, G12, G13

Acknowledgements: I would like to thank Professor Moisă Altăr for continuous support. 


\section{INTRODUCTION}

In the context of the classical models for valuing options (Black and Scholes, 1973; Merton, 1973a) the derivative is redundant since it can be replicated using other existing assets (i.e. the underlying and the risk-free bond). These market models are complete so that there is only one risk neutral measure and consequently only one fair price of the derivative. The disadvantage of these models is the assumption that the returns are normally distributed. To account for the high kurtosis of the empirical distribution of returns, several models based on Levy processes with jumps were developed. But in these cases the market models are not complete and the risk neutral measure is not unique. Therefore it is difficult to compute the fair price of the derivative. A solution of this problem is to evaluate the derivative in the context of a general equilibrium model taking explicitly into account the risk premium.

In this paper we develop a framework for asset pricing in the context of the general equilibrium model in Necula (2008). We analyze a benchmark specification of this model consisting of constant growth rates and volatilities for the output and the money supply, and of log-normal amplitude of the jumps. Using this specification we deduce the fundamental evaluation equations for financial assets, the dynamics of the real and of the nominal exchange rates and a new formula for evaluating exchange rate options.

This paper is organized as follows: in the second section we present the hypotheses of the benchmark model and we obtain the probability distribution of the nominal exchange rate. In the third section we focus on the fundamental evaluation equations for different types of assets. In the forth section we deal with the particular case of currency derivatives and obtain a new formula for pricing exchange rate options. The final section concludes.

\section{THE MODEL}

We consider a benchmark economy build upon the framework developed in Necula (2008). The two goods utility function is separable consisting of the sum of two CRRA one good utility functions with different risk aversion parameters:

$$
U\left(c, c^{*}\right)=\frac{c^{1-\gamma}}{1-\gamma}+\frac{c^{1-\gamma^{*}}}{1-\gamma^{*}}
$$

The growth rate and the volatility of the output are constant: 


$$
\frac{d y_{t}}{y_{t}}=\left(\mu_{y}-\lambda E[J]\right) d t+\sigma_{y} d \omega_{y t}+J d N_{t}
$$

where the jumps amplitude is log-normally distributed:

$$
\ln (1+J) \sim \operatorname{Norm}\left(\mu_{J}, \sigma_{J}^{2}\right)
$$

The growth rate and the volatility of the money supply are also constant. To keep the model parsimonious we assume that the GDP and the money supply are not correlated:

$$
\frac{d m_{t}}{m_{t}}=\mu_{m} d t+\sigma_{m} d \omega_{m t}, d \omega_{m t} d \omega_{y t}=0
$$

We also assume that there is no correlation between variables across the two countries:

$$
d \omega_{y} d \omega_{y}^{*}=0, d \omega_{m} d \omega_{m}^{*}=0
$$

The following notations will be used:

$$
\begin{gathered}
a_{1}(\gamma):=\gamma \mu_{y}-\frac{\gamma(\gamma+1)}{2} \sigma_{y}^{2}-\lambda\left[\gamma \exp \left(\mu_{J}+\frac{\sigma_{J}^{2}}{2}\right)+\exp \left(-\gamma \mu_{J}+\gamma^{2} \frac{\sigma_{J}^{2}}{2}\right)-(\gamma+1)\right] \\
a_{2}(\gamma):=\lambda\left[\exp \left(-\gamma \mu_{J}+\gamma^{2} \frac{\sigma_{J}^{2}}{2}\right)-1\right]
\end{gathered}
$$

Proposition 1. In the economy characterized by the equations (1)-(5) we have that:

a) the real interest rate is constant:

$$
r=\phi+a_{1}(\gamma)
$$

b) the nominal interest rate is constant:

$$
R=\phi+a_{1}(\gamma-1)+\mu_{m}-\sigma_{m}^{2}
$$

c) the real price of a Home equity share:

$$
s_{t}=\frac{2 y_{t}}{\phi+a_{1}(\gamma-1)}
$$

d) the dynamics of the real exchange rate is: 


$$
\begin{aligned}
\frac{d \varepsilon_{t}}{\varepsilon_{t}} & =\left[\left(r-r^{*}\right)+\gamma^{2} \sigma_{y}^{2}+a_{2}(\gamma)-a_{2}^{*}\left(\gamma^{*}\right)\right] d t \\
& +\gamma \sigma_{y} d \omega_{y}-\gamma^{*} \sigma_{y}^{*} d \omega_{y}^{*}+\left[(1+J)^{\gamma}-1\right] d N_{t}+\left[\left(1+J^{*}\right)^{-\gamma^{*}}-1\right] d N_{t}^{*}
\end{aligned}
$$

e) the real exchange rate at $T$ is given by:

$$
\begin{aligned}
\ln \varepsilon_{T} & =\ln \varepsilon_{t}+\left\{\left(r-r^{*}\right)+\frac{\gamma^{2} \sigma_{y}^{2}}{2}-\frac{\gamma^{* 2} \sigma_{y}^{* 2}}{2}+a_{2}(\gamma)-a_{2}^{*}\left(\gamma^{*}\right)\right\}(T-t) \\
& +\gamma \sigma_{y}\left[\omega_{y}(T)-\omega_{y}(t)\right]-\gamma^{*} \sigma_{y}^{*}\left[\omega_{y}^{*}(T)-\omega_{y}^{*}(t)\right]+\gamma \sum_{i=N_{t}+1}^{N_{T}} \ln \left(1+J_{i}\right)-\gamma^{*} \sum_{i=N_{t}^{*}+1}^{N_{T}^{*}} \ln \left(1+J_{i}^{*}\right)
\end{aligned}
$$

f) the dynamics of the nominal exchange rate is:

$$
\begin{aligned}
\frac{d e_{t}}{e_{t}}= & {\left[\left(R-R^{*}\right)+\sigma_{m}^{2}+(\gamma-1)^{2} \sigma_{y}^{2}+a_{2}(\gamma-1)-a_{2}^{*}\left(\gamma^{*}-1\right)\right] d t } \\
& +(\gamma-1) \sigma_{y} d \omega_{y}+\sigma_{m} d \omega_{m}-\left(\gamma^{*}-1\right) \sigma_{y}^{*} d \omega_{y}^{*}-\sigma_{m}^{*} d \omega_{m}^{*} \\
& +\left[(1+J)^{\gamma-1}-1\right] d N_{t}+\left[\left(1+J^{*}\right)^{-\left(\gamma^{*}-1\right)}-1\right] d N_{t}^{*}
\end{aligned}
$$

g) the nominal exchange rate at $T$ is given by:

$$
\begin{aligned}
\ln e_{T} & =\ln e_{t}+\left\{\left(R-R^{*}\right)+\frac{(\gamma-1)^{2} \sigma_{y}^{2}+\sigma_{m}^{2}}{2}-\frac{\left(\gamma^{*}-1\right)^{2} \sigma_{y}^{* 2}+\sigma_{m}^{* 2}}{2}+a_{2}(\gamma-1)-a_{2}^{*}\left(\gamma^{*}-1\right)\right\}(T-t) \\
& +(\gamma-1) \sigma_{y}\left[\omega_{y}(T)-\omega_{y}(t)\right]-\left(\gamma^{*}-1\right) \sigma_{y}^{*}\left[\omega_{y}^{*}(T)-\omega_{y}^{*}(t)\right] \\
& +\sigma_{m}\left[\omega_{m}(T)-\omega_{m}(t)\right]-\sigma_{m}^{*}\left[\omega_{m}^{*}(T)-\omega_{m}^{*}(t)\right] \\
& +(\gamma-1) \sum_{i=N_{t}+1}^{N_{T}} \ln \left(1+J_{i}\right)-\left(\gamma^{*}-1\right) \sum_{i=N_{t}^{*}+1}^{N_{T}^{*}} \ln \left(1+J_{i}^{*}\right)
\end{aligned}
$$

\section{Proof:}

Equations (8), (9) and (10) follow from Proposition 1 in Necula (2008). The price of the Home equity share is consistent with Naik and Lee (1990) that use a one country model with jumps in GDP.

The dynamics in (11) and (13) follow Proposition 4 in Necula (2008).

Equation (12) and (14) are a straightforward application of the Ito Lemma for $\ln \varepsilon$, and $\ln e$ respectively. 
It is important to point out that in the case of a logarithmic utility function (i.e. $\gamma=\gamma^{*}=1$ ) there are no jumps in the nominal exchange rate and the dynamics is influenced exclusively by the money supply.

Proposition 2. In the economy characterized by the equations (1)-(5) we have that the distribution of the log return of the nominal exchange rate is a mixture of normal distributions with a pdf given by:

$$
\varphi_{\ln e_{T}-\ln e_{t}}(x)=\sum_{n} \sum_{n^{*}} p(n) p^{*}\left(n^{*}\right) \varphi_{n, n^{*}}(x)
$$

where $\varphi_{n, n^{*}}$ is the pdf of a normal distribution

$$
\operatorname{Norm}\left(\left(\mu_{n}-\bar{\mu}_{n^{*}}\right)(T-t),\left(\sigma_{n}^{2}+\bar{\sigma}_{n^{*}}^{2}\right)(T-t)\right)
$$

with

$$
\begin{aligned}
& \mu_{n}=R+\frac{(\gamma-1)^{2} \sigma_{y}^{2}+\sigma_{m}^{2}}{2}+a_{2}(\gamma-1)+\frac{n}{T-t}(\gamma-1) \mu_{J} \\
& \bar{\mu}_{n^{*}}=R^{*}+\frac{\left(\gamma^{*}-1\right)^{2} \sigma_{y}^{* 2}+\sigma_{m}^{* 2}}{2}+a_{2}^{*}\left(\gamma^{*}-1\right)+\frac{n^{*}}{T-t}\left(\gamma^{*}-1\right) \mu_{J}^{*}
\end{aligned}
$$

and

$$
\begin{aligned}
& \sigma_{n}^{2}=(\gamma-1)^{2}\left(\sigma_{y}^{2}+\frac{n}{T-t} \sigma_{J}^{2}\right)+\sigma_{m}^{2} \\
& \bar{\sigma}_{n^{*}}^{2}=\left(\gamma^{*}-1\right)^{2}\left(\sigma_{y}^{* 2}+\frac{n^{*}}{T-t} \sigma_{J}^{* 2}\right)+\sigma_{m}^{* 2}
\end{aligned}
$$

\section{Proof:}

From equation (14) one can observe the distribution of $\ln \left(e_{T}\right)-\ln \left(e_{t}\right)$ conditioned by the fact that there were $n$ Home jumps and $n^{*}$ Foreign jumps is given by:

$$
\ln \frac{e_{T}}{e_{t}} \mid N_{T}-N_{t}=n, N_{T}^{*}-N_{t}^{*}=n^{*} \sim \operatorname{Norm}\left(\left(\mu_{n}-\bar{\mu}_{n^{*}}\right)(T-t),\left(\sigma_{n}^{2}+\bar{\sigma}_{n^{*}}^{2}\right)(T-t)\right)
$$

Equation (15) is a straightforward application of Bayes Theorem. 


\section{THE FUNDAMENTAL ASSET PRICING EQUATIONS}

Proposition 3. In the economy characterized by the equations (1)-(5) we have the following fundamental asset pricing equations:

a) if the asset pays real dividends depending only on the Home output (i.e. $\delta(y)$ ) then the real price $q(t, y)$ of the asset is the solution of the PIDE:

$$
\begin{aligned}
\frac{\partial q}{\partial t}+\delta(y)+\left(\mu_{y}-\lambda E[J]-\gamma \sigma_{y}^{2}\right) y \frac{\partial q}{\partial y}+\frac{1}{2} \sigma_{y}^{2} y^{2} \frac{\partial^{2} q}{\partial y^{2}} \\
+\lambda \int_{\mathbf{R}} \frac{q(y(1+x))-q(y)}{(1+x)^{\gamma}} \varphi_{J}(x) d x=r q
\end{aligned}
$$

b) if the asset pays real dividends depending both on the Home output and of the Foreign output (i.e. $\delta\left(y, y^{*}, \varepsilon\right)$ ) then the real price $q\left(t, y, y^{*}, \varepsilon\right)$ of the asset is the solution of the PIDE:

$$
\begin{aligned}
& \frac{\partial q}{\partial t}+\delta\left(y, y^{*}, \varepsilon\right)+\left(\mu_{y}-\lambda E[J]-\gamma \sigma_{y}^{2}\right) y \frac{\partial q}{\partial y}+\left(\mu_{y}^{*}-\lambda^{*} E\left[J^{*}\right]\right) y^{*} \frac{\partial q}{\partial y^{*}} \\
& +\left[\left(r-r^{*}\right)+a_{2}(\gamma)-a_{2}^{*}\left(\gamma^{*}\right)\right] \varepsilon \frac{\partial q}{\partial \varepsilon} \\
& +\frac{1}{2} \sigma_{y}^{2} y^{2} \frac{\partial^{2} q}{\partial y^{2}}+\frac{1}{2} \sigma_{y}^{* 2} y^{* 2} \frac{\partial^{2} q}{\partial y^{* 2}}+\frac{1}{2}\left(\gamma^{2} \sigma_{y}^{2}+\gamma^{* 2} \sigma_{y}^{* 2}\right) \varepsilon^{2} \frac{\partial^{2} q}{\partial \varepsilon^{2}} \\
& +\gamma \sigma_{y}^{2} y \varepsilon \frac{\partial^{2} q}{\partial y \partial \varepsilon}-\gamma^{*} \sigma_{y}^{* 2} y^{*} \varepsilon \frac{\partial^{2} q}{\partial y^{*} \partial \varepsilon} \\
& +\lambda \int_{\mathbf{R}} \frac{q\left(y(1+x), y^{*}, \varepsilon(1+x)^{\gamma}\right)-q\left(y, y^{*}, \varepsilon\right)}{(1+x)^{\gamma}} \varphi_{J}(x) d x \\
& +\lambda^{*}\left[q\left(y, y_{\mathbf{R}}^{*}(1+x), \varepsilon(1+x)^{-\gamma^{*}}\right)-q\left(y, y^{*}, \varepsilon\right)\right] \varphi_{J^{*}}(x) d x=r q
\end{aligned}
$$

c) if the asset pays nominal dividends depending only on the Home variables (i.e. $\Gamma(y, m))$ then the nominal price $Q(t, y, m)$ of the asset is the solution of the PIDE: 


$$
\begin{aligned}
\frac{\partial Q}{\partial t}+\Gamma(y, m)+\left(\mu_{y}-\lambda E[J]-\right. & \left.(\gamma-1) \sigma_{y}^{2}\right) y \frac{\partial Q}{\partial y}+\left(\mu_{m}-\sigma_{m}^{2}\right) m \frac{\partial Q}{\partial m} \\
& +\frac{1}{2} \sigma_{y}^{2} y^{2} \frac{\partial^{2} q}{\partial y^{2}}+\frac{1}{2} \sigma_{m}^{2} m^{2} \frac{\partial^{2} q}{\partial m^{2}} \\
& +\lambda \int_{\mathbf{R}} \frac{Q(y(1+x), m)-Q(y, m)}{(1+x)^{\gamma-1}} \varphi_{J}(x) d x=R Q
\end{aligned}
$$

d) if the asset pays nominal dividends depending both on the Home and Foreign variables (i.e. $\Gamma\left(y, m, y^{*}, m^{*}, e\right)$ ) then the nominal price $Q\left(t, y, m, y^{*}, m^{*}, e\right)$ of the asset is the solution of the PIDE:

$$
\begin{aligned}
& \frac{\partial Q}{\partial t}+\Gamma\left(y, m, y^{*}, m^{*}, e\right)+\left(\mu_{y}-\lambda E[J]-(\gamma-1) \sigma_{y}^{2}-\sigma_{m}^{2}\right) y \frac{\partial Q}{\partial y}+\left(\mu_{y}^{*}-\lambda^{*} E\left[J^{*}\right]\right) y^{*} \frac{\partial Q}{\partial y^{*}} \\
& +\left(\mu_{m}-\sigma_{m}^{2}\right) m \frac{\partial Q}{\partial m}+\mu_{m}^{*} m^{*} \frac{\partial Q}{\partial m^{*}}+\left[\left(R-R^{*}\right)+a_{2}(\gamma-1)-a_{2}^{*}\left(\gamma^{*}-1\right)\right] e \frac{\partial Q}{\partial e} \\
& +\frac{1}{2} \sigma_{y}^{2} y^{2} \frac{\partial^{2} Q}{\partial y^{2}}+\frac{1}{2} \sigma_{y}^{* 2} y^{* 2} \frac{\partial^{2} Q}{\partial y^{* 2}}+\frac{1}{2} \sigma_{y}^{2} m^{2} \frac{\partial^{2} Q}{\partial m^{2}}+\frac{1}{2} \sigma_{m}^{* 2} m^{* 2} \frac{\partial^{2} Q}{\partial m^{* 2}} \\
& +\frac{1}{2}\left[(\gamma-1)^{2} \sigma_{y}^{2}+\left(\gamma^{*}-1\right)^{2} \sigma_{y}^{* 2} e^{2} \frac{\partial^{2} Q}{\partial e^{2}}\right. \\
& +(\gamma-1) \sigma_{y}^{2} y e \frac{\partial^{2} Q}{\partial y \partial e}+\sigma_{m}^{2} m e \frac{\partial^{2} Q}{\partial m \partial e}-\left(\gamma^{*}-1\right) \sigma_{y}^{* 2} y^{*} e \frac{\partial^{2} Q}{\partial y^{*} \partial e}-\sigma_{m}^{* 2} m^{*} e \frac{\partial^{2} Q}{\partial m^{*} \partial e} \\
& +\lambda \int_{\mathbf{R}} \frac{Q\left(y(1+x), m, y^{*}, m^{*}, e(1+x)^{\gamma-1}\right)-Q\left(y, m, y^{*}, m^{*}, e\right)}{(1+x)^{\gamma-1}} \varphi_{J}(x) d x \\
& +\lambda^{*}\left[Q\left[Q\left(y, m, y^{*}(1+x), m^{*}, e(1+x)^{-\left(\gamma^{*}-1\right)}\right)-Q\left(y, m, y^{*}, m^{*}, e\right)\right] \varphi_{J^{*}}(x) d x=R Q\right.
\end{aligned}
$$

\section{Proof:}

Using Ito Lemma for $q(t, y)$ we get the following dynamics for the asset price:

$$
d q=\frac{\partial q}{\partial t} d t+\frac{\partial q}{\partial y} y\left(\frac{d y}{y}\right)_{c}+\frac{1}{2} \frac{\partial^{2} q}{\partial y^{2}} y^{2}\left(\frac{d y}{y}\right)_{c}^{2}+[q(y(1+J))-q(y)] d N
$$

From Proposition 3 in Necula (2008) we have that the expected return is given by: 


$$
\frac{1}{d t} E_{t}\left[\frac{d q+\delta(y) d t}{q}\right]=\mu_{q}=r+\gamma \frac{y}{q} \frac{\partial q}{\partial y} \sigma_{y}^{2}-\lambda E\left\{\frac{q(y(1+J))-q(y)}{q(y)}\left[(1+J)^{-\gamma}-1\right]\right\}
$$

Equation (19) follows from (23) and (24).

If the asset pays real dividends depending both on the Home output and of the Foreign output we apply the Ito Lemma to the function $q\left(t, y, y^{*}, \varepsilon\right)$ :

$$
\begin{aligned}
d q & =\frac{\partial q}{\partial t} d t+\frac{\partial q}{\partial y} y\left(\frac{d y}{y}\right)_{c}+\frac{\partial q}{\partial y^{*}} y^{*}\left(\frac{d y^{*}}{y^{*}}\right)_{c}+\frac{\partial q}{\partial \varepsilon} \varepsilon\left(\frac{d \varepsilon}{\varepsilon}\right)_{c} \\
& +\frac{1}{2} \frac{\partial^{2} q}{\partial y^{2}} y^{2}\left(\frac{d y}{y}\right)_{c}^{2}+\frac{1}{2} \frac{\partial^{2} q}{\partial y^{* 2}} y^{* 2}\left(\frac{d y^{*}}{y^{*}}\right)_{c}^{2}+\frac{1}{2} \frac{\partial^{2} q}{\partial \varepsilon^{2}} \varepsilon^{2}\left(\frac{d \varepsilon}{\varepsilon}\right)_{c}^{2} \\
& +\frac{\partial^{2} q}{\partial y \partial \varepsilon} y \varepsilon\left(\frac{d y}{y}\right)_{c}\left(\frac{d \varepsilon}{\varepsilon}\right)_{c}+\frac{\partial^{2} q}{\partial y^{*} \partial \varepsilon} y^{*} \varepsilon\left(\frac{d y^{*}}{y^{*}}\right)_{c}\left(\frac{d \varepsilon}{\varepsilon}\right)_{c} \\
& +\left[q\left(y(1+J), y^{*}, \varepsilon(1+J)^{\gamma}\right)-q\left(y, y^{*}, \varepsilon\right)\right] d N \\
& +\left[q\left(y, y^{*}\left(1+J^{*}\right), \varepsilon\left(1+J^{*}\right)^{-\gamma^{*}}\right)-q\left(y, y^{*}, \varepsilon\right)\right] d N^{*}
\end{aligned}
$$

In order to get equation (20), we use the results from Proposition 3 in Necula (2008) regarding the expected return:

$$
\begin{aligned}
\mu_{q} & =r+\gamma \frac{y}{q} \frac{\partial q}{\partial y} \sigma_{y}^{2}+\gamma^{2} \frac{\varepsilon}{q} \frac{\partial q}{\partial e} \sigma_{y}^{2} \\
& -\lambda E\left\{\frac{q\left(y(1+J), y^{*}, \varepsilon(1+J)^{\gamma}\right)-q\left(y, y^{*}, \varepsilon\right)}{q\left(y, y^{*}, \varepsilon\right)}\left[(1+J)^{-\gamma}-1\right]\right\}
\end{aligned}
$$

If the asset pays nominal dividends depending only on the Home variables we use the Ito Lemma to a function of the form $Q(t, y, m)$ :

$$
\begin{aligned}
d Q=\frac{\partial Q}{\partial t} d t+\frac{\partial Q}{\partial y} y\left(\frac{d y}{y}\right)_{c}+\frac{\partial Q}{\partial m} m\left(\frac{d m}{m}\right)_{c}+ & \frac{1}{2} \frac{\partial^{2} Q}{\partial y^{2}} y^{2}\left(\frac{d y}{y}\right)_{c}^{2}+\frac{1}{2} \frac{\partial^{2} Q}{\partial m^{2}} m^{2}\left(\frac{d m}{m}\right)_{c}^{2} \\
& +[q(y(1+J), m)-q(y, m)] d N
\end{aligned}
$$

Again from Proposition 3 in Necula (2008) the nominal expected return is: 


$$
\mu_{Q}=R+(\gamma-1) \frac{y}{Q} \frac{\partial Q}{\partial y} \sigma_{y}^{2}+\frac{m}{Q} \frac{\partial Q}{\partial m} \sigma_{m}^{2}-\lambda E\left\{\frac{q(y(1+J), m)-q(y, m)}{q(y, m)}\left[(1+J)^{-\gamma}-1\right]\right\}
$$

If the asset pays nominal dividends depending both on the Home and Foreign variables we use Ito Lemma for a function $Q\left(t, y, m, y^{*}, m^{*}, e\right)$ :

$$
\begin{aligned}
d Q & =\frac{\partial Q}{\partial t} d t+\frac{\partial Q}{\partial y} y\left(\frac{d y}{y}\right)_{c}+\frac{\partial Q}{\partial y^{*}} y^{*}\left(\frac{d y^{*}}{y^{*}}\right)_{c}+\frac{\partial Q}{\partial m} m\left(\frac{d m}{m}\right)_{c}+\frac{\partial Q}{\partial m^{*}} m^{*}\left(\frac{d m^{*}}{m^{*}}\right)_{c} \\
& +\frac{\partial Q}{\partial e} e\left(\frac{d e}{e}\right)_{c}+\frac{1}{2} \frac{\partial^{2} Q}{\partial y^{2}} y^{2}\left(\frac{d y}{y}\right)_{c}^{2}+\frac{1}{2} \frac{\partial^{2} Q}{\partial y^{* 2}} y^{* 2}\left(\frac{d y^{*}}{y^{*}}\right)_{c}^{2}+\frac{1}{2} \frac{\partial^{2} Q}{\partial e^{2}} e^{2}\left(\frac{d e}{e}\right)_{c}^{2} \\
& +\frac{1}{2} \frac{\partial^{2} Q}{\partial m^{2}} m^{2}\left(\frac{d m}{m}\right)_{c}^{2}+\frac{1}{2} \frac{\partial^{2} Q}{\partial m^{* 2}} m^{* 2}\left(\frac{d y^{*}}{y^{*}}\right)_{c}^{2} \\
& +\frac{\partial^{2} Q}{\partial y \partial e} y e\left(\frac{d y}{y}\right)_{c}\left(\frac{d e}{e}\right)_{c}+\frac{\partial^{2} Q}{\partial y^{*} \partial e^{*}} y^{*} e\left(\frac{d y^{*}}{y^{*}}\right)_{c}\left(\frac{d e}{e}\right)_{c} \\
& +\frac{\partial^{2} Q}{\partial m \partial e} m e\left(\frac{d m}{m}\right)_{c}\left(\frac{d e}{e}\right)_{c}+\frac{\partial^{2} q}{\partial y^{*} \partial e} y^{*} e\left(\frac{d y^{*}}{y^{*}}\right)_{c}\left(\frac{d e}{e}\right)_{c} \\
& +\left[Q\left(y(1+J), m, y^{*}, m^{*}, e(1+J)^{(\gamma-1)}\right)-Q\left(y, m, y^{*}, m^{*}, e\right)\right] d N \\
& +\left[Q\left(y, m, y^{*}\left(1+J^{*}\right), m^{*}, e\left(1+J^{*}\right)^{-\left(y^{*}-1\right)}\right)_{-Q} Q\left(y, m, y^{*}, m^{*}, e\right)\right] d N^{*}
\end{aligned}
$$

In equilibrium the expected return is (Proposition 3 in Necula, 2008):

$$
\begin{aligned}
\mu_{Q} & =R+(\gamma-1) \frac{y}{Q} \frac{\partial Q}{\partial y} \sigma_{y}^{2}+(\gamma-1)^{2} \frac{e}{Q} \frac{\partial Q}{\partial e} \sigma_{y}^{2}+\frac{m}{Q} \frac{\partial Q}{\partial m} \sigma_{m}^{2}+\frac{e}{Q} \frac{\partial Q}{\partial e} \sigma_{m}^{2} \\
& -\lambda E\left\{\frac{Q\left(y(1+J), m, y^{*}, m^{*}, e(1+J)^{(\gamma-1)}\right)-Q\left(y, m, y^{*}, m^{*}, \varepsilon\right)}{Q\left(y, m, y^{*}, m^{*}, \varepsilon\right)}\left[(1+J)^{-(\gamma-1)}-1\right]\right\}
\end{aligned}
$$

q.e.d.

It is straightforward to prove that if the asset pays a cash-flow $\Gamma\left(y, m, y^{*}, m^{*}, e\right)=\Gamma_{H}(y, m)+e \Gamma_{F}\left(y^{*}, m^{*}\right)$, then the price of the financial asset is given by $Q\left(t, y, m, y^{*}, m^{*}, e\right)=Q_{H}(t, y, m)+e Q_{F}\left(t, y^{*}, m^{*}\right)$, where $Q_{H}$ and $Q_{F}$ are the solutions of the equation (21) written for Home, and Foreign respectively. 


\section{PRICING EXCHANGE RATE OPTIONS}

Consider a call exchange rate option with maturity $T=t+\tau$ and strike price $K$. Using Corollary 1 in Necula (2008), the price of this contingent claim is:

$$
C_{t}=C\left(e_{t}, y_{t}, y_{t}^{*}, m_{t}, m_{t}^{*}\right)=E_{t}\left[\exp (-\phi \tau) \frac{y_{T} u^{\prime}\left(y_{T}\right)}{y_{t} u^{\prime}\left(y_{t}\right)} \frac{m_{t}}{m_{T}} \max \left(e_{T}-K, 0\right)\right]
$$

Proposition 4. In the economy characterized by the equations (1)-(5) the price of an exchange rate call option is given by:

$$
C_{t}=\sum_{n} \sum_{n^{*}} p(n) p^{*}\left(n^{*}\right) C_{n, n^{*}}
$$

where

$$
\begin{gathered}
C_{n, n^{*}}=e_{t} \exp \left\{-\left(\bar{\mu}_{n^{*}}-\frac{\bar{\sigma}_{n^{*}}^{2}}{2}\right) \tau\right\} \Phi\left(d_{1, n, n^{*}}\right)-K \exp \left\{-\left(\mu_{n}-\frac{\sigma_{n}^{2}}{2}\right) \tau\right\} \Phi\left(d_{2, n, n^{*}}\right) \\
d_{1, n, n^{*}}=\frac{\ln \frac{e_{t}}{K}+\left(\mu_{n}-\bar{\mu}_{n^{*}}+\bar{\sigma}_{n}^{2}\right) \tau}{\sqrt{\sigma_{n}^{2}+\bar{\sigma}_{n^{*}}^{2}} \sqrt{\tau}} \\
d_{2, n, n^{*}}=\frac{\ln \frac{e_{t}}{K}+\left(\mu_{n}-\bar{\mu}_{n^{*}}+\sigma_{n}^{2}\right) \tau}{\sqrt{\sigma_{n}^{2}+\bar{\sigma}_{n^{*}}^{2}} \sqrt{\tau}}
\end{gathered}
$$

and $\Phi(\cdot)$ is the cdf of the standard normal distribution.

\section{Proof:}

Using Bayes Theorem the equation (31) can be written:

$$
C_{t}=\sum_{n} \sum_{n^{*}} p(n) p^{*}\left(n^{*}\right) C_{n, n^{*}}
$$

where 


$$
\begin{aligned}
C_{n, n^{*}}= & E_{t}\left[\exp (-\phi \tau) \frac{y_{T} u^{\prime}\left(y_{T}\right)}{y_{t} u^{\prime}\left(y_{t}\right)} \frac{m_{t}}{m_{T}} \max \left(e_{T}-K, 0\right) \mid N_{T}-N_{t}=n, N_{T}^{*}-N_{t}^{*}=n^{*}\right] \\
= & E_{t}\left[\exp (-\phi \tau) \frac{y_{T} u^{\prime}\left(y_{T}\right)}{y_{t} u^{\prime}\left(y_{t}\right)} \frac{m_{t}}{m_{T}} e_{T} 1_{e_{T} \geq K} \mid N_{T}-N_{t}=n, N_{T}^{*}-N_{t}^{*}=n^{*}\right] \\
& -K E_{t}\left[\exp (-\phi \tau) \frac{y_{T} u^{\prime}\left(y_{T}\right)}{y_{t} u^{\prime}\left(y_{t}\right)} \frac{m_{t}}{m_{T}} 1_{e_{T} \geq K} \mid N_{T}-N_{t}=n, N_{T}^{*}-N_{t}^{*}=n^{*}\right]
\end{aligned}
$$

Using equations (14) and (18) the nominal exchange rate at $T$ is given by:

$$
e_{T}=e_{t} \exp \left\{\left(\mu_{n}-\bar{\mu}_{n}\right) \tau\right\} \exp \left\{\left(\sigma_{n} X-\bar{\sigma}_{n^{*}} \bar{X}\right) \sqrt{\tau}\right\}
$$

where

$$
X, \bar{X} \text { i.i.d. } \operatorname{Norm}(0,1)
$$

We also have that:

$$
\exp (-\phi \tau) \frac{y_{T} u^{\prime}\left(y_{T}\right)}{y_{t} u^{\prime}\left(y_{t}\right)} \frac{m_{t}}{m_{T}}=\exp \left(-\mu_{n} \tau\right) \exp \left(-\sigma_{n} X \sqrt{\tau}\right)
$$

We denote:

$$
Z:=\frac{\sigma_{n} X-\bar{\sigma}_{n}{ }^{*} \bar{X}}{\sqrt{\sigma_{n}^{2}+\bar{\sigma}_{n^{*}}^{2}}} \sim \operatorname{Norm}(0,1)
$$

The nominal exchange rate at the maturity of the option is:

$$
e_{T}=e_{t} \exp \left\{\left(\mu_{n}-\bar{\mu}_{n}\right) \tau\right\} \exp \left\{\sqrt{\sigma_{n}^{2}+\bar{\sigma}_{n}^{2}} Z \sqrt{\tau}\right\}
$$

First we evaluate the following conditional expectation:

$$
\begin{gathered}
E_{t}\left[\exp \{-\phi(T-t)\} \frac{y_{T} u^{\prime}\left(y_{T}\right)}{y_{t} u^{\prime}\left(y_{t}\right)} \frac{m_{t}}{m_{T}} 1_{e_{T} \geq K} \mid N_{T}-N_{t}=n, N_{T}^{*}-N_{t}^{*}=n^{*}\right]= \\
=\exp \left(-\mu_{n} \tau\right) E\left[\exp \left(-\sigma_{n} X \sqrt{\tau}\right) \boldsymbol{h}_{Z \geq-\alpha}\right]
\end{gathered}
$$

where 


$$
\alpha=\frac{\ln \frac{e_{t}}{K}+\left(\mu_{n}-\bar{\mu}_{n}\right) \tau}{\sqrt{\sigma_{n}^{2}+\bar{\sigma}_{n^{*}}^{2}} \sqrt{\tau}}
$$

It is obvious that:

$$
(X, Z) \sim \mathrm{Norm}_{2}\left(\left(\begin{array}{l}
0 \\
0
\end{array}\right), \cdot\left(\begin{array}{ll}
1 & \rho \\
\rho & 1
\end{array}\right)\right)
$$

where

$$
\rho=\frac{\sigma_{n}}{\sqrt{\sigma_{n}^{2}+\bar{\sigma}_{n^{*}}^{2}}}
$$

It follows that:

$$
\begin{gathered}
E\left[\exp \left(-\sigma_{n} X \sqrt{\tau}\right) \mathrm{l}_{z \geq-\alpha}\right\rfloor \\
=\frac{1}{2 \pi \sqrt{\left(1-\rho^{2}\right)}} \int_{-\infty}^{\infty} \int_{-\alpha}^{\infty} \exp \left(-\sigma_{n} \sqrt{\tau} x\right) \exp \left(-\frac{1}{2} \frac{x^{2}-2 \rho x y+y^{2}}{1-\rho^{2}}\right) d x d y \\
=\int_{-\alpha}^{\infty} \frac{1}{\sqrt{2 \pi}} \exp \left(-\frac{1}{2} y^{2}\right) \int_{-\infty}^{\infty} \frac{1}{\sqrt{2 \pi\left(1-\rho^{2}\right)}} \exp \left(-\sigma_{n} \sqrt{\tau} x\right) \exp \left(-\frac{1}{2} \frac{x^{2}-2 \rho x y+\rho^{2} y^{2}}{1-\rho^{2}}\right) d x d y
\end{gathered}
$$

But,

$$
\begin{aligned}
& \exp \left(-\sigma_{n} \sqrt{\tau} x\right) \exp \left(-\frac{1}{2} \frac{x^{2}-2 \rho x y-\rho^{2} y^{2}}{1-\rho^{2}}\right) \\
& =\exp \left\{-\frac{1}{2}\left[\left(\frac{x}{\sqrt{1-\rho^{2}}}\right)^{2}+2 \sigma_{n} \sqrt{\tau} x-2 \frac{x}{\sqrt{1-\rho^{2}}} \frac{\rho y}{\sqrt{1-\rho^{2}}}+\left(\frac{\rho y}{\sqrt{1-\rho^{2}}}\right)^{2}\right]\right\}
\end{aligned}
$$

On the other hand: 


$$
\begin{aligned}
& \left(\frac{x}{\sqrt{1-\rho^{2}}}\right)^{2}+2 \sigma_{n} \sqrt{\tau} x-2 \frac{x}{\sqrt{1-\rho^{2}}} \frac{\rho y}{\sqrt{1-\rho^{2}}}+\left(\frac{\rho y}{\sqrt{1-\rho^{2}}}\right)^{2} \\
& =\left(\frac{x}{\sqrt{1-\rho^{2}}}\right)^{2}+2 \sigma_{n} \sqrt{\tau} x+\sigma_{n}^{2} \tau\left(1-\rho^{2}\right)-\sigma_{n}^{2} \tau\left(1-\rho^{2}\right) \\
& -2\left(\frac{x}{\sqrt{1-\rho^{2}}}+\sigma_{n} \sqrt{\tau} \sqrt{1-\rho^{2}}-\sigma_{n} \sqrt{\tau} \sqrt{1-\rho^{2}}\right) \frac{\rho y}{\sqrt{1-\rho^{2}}}+\left(\frac{\rho y}{\sqrt{1-\rho^{2}}}\right)^{2} \\
& =\left(\frac{x}{\sqrt{1-\rho^{2}}}+\sigma_{n} \sqrt{\tau} \sqrt{1-\rho^{2}}\right)^{2}-2\left(\frac{x}{\sqrt{1-\rho^{2}}}+\sigma_{n} \sqrt{\tau} \sqrt{1-\rho^{2}}\right) \frac{\rho y}{\sqrt{1-\rho^{2}}}+\left(\frac{\rho y}{\sqrt{1-\rho^{2}}}\right)^{2} \\
& =\left(\frac{x}{\sqrt{1-\rho^{2}}}+\sigma_{n} \sqrt{\tau} \sqrt{1-\rho^{2}}-\frac{\rho y}{\sqrt{1-\rho^{2}}}\right)^{2}+2 \rho \sigma_{n} \sqrt{\tau} y-\sigma_{n}^{2} \tau\left(1-\rho^{2}\right)
\end{aligned}
$$

But it is straightforward that:

$$
\int_{-\infty}^{\infty} \frac{1}{\sqrt{2 \pi\left(1-\rho^{2}\right)}} \exp \left\{-\frac{1}{2}\left(\frac{x}{\sqrt{1-\rho^{2}}}+\sigma_{n} \sqrt{\tau} \sqrt{1-\rho^{2}}-\frac{\rho y}{\sqrt{1-\rho^{2}}}\right)^{2}\right\} d x=1
$$

We have that:

$$
\begin{aligned}
E\left[\exp \left(-\sigma_{n} X \sqrt{\tau}\right) \mathbb{1}_{Z \geq-\alpha}\right] & =\exp \left(\frac{1}{2} \sigma_{n}^{2} \tau\left(1-\rho^{2}\right)\right) \int_{-\alpha}^{\infty} \frac{1}{\sqrt{2 \pi}} \exp \left(-\frac{1}{2} y^{2}-\rho \sigma_{n} \sqrt{\tau} y\right) d y \\
& =\exp \left(\frac{1}{2} \sigma_{n}^{2} \tau\right) \int_{-\alpha}^{\infty} \frac{1}{\sqrt{2 \pi}} \exp \left\{-\frac{1}{2}\left(y+\rho \sigma_{n} \sqrt{\tau}\right)^{2}\right\} d y \\
& =\exp \left(\frac{1}{2} \sigma_{n}^{2} \tau\right) \Phi\left(d_{2, n, n^{*}}\right)
\end{aligned}
$$

Analogously, in order to evaluate: 


$$
\begin{array}{r}
E_{t}\left[\exp (-\phi \tau) e_{T} \frac{y_{T} u^{\prime}\left(y_{T}\right)}{y_{t} u^{\prime}\left(y_{t}\right)} \frac{m_{t}}{m_{T}} 1_{e_{T} \geq K} \mid N_{T}-N_{t}=n, N_{T}^{*}-N_{t}^{*}=n^{*}\right] \\
=\exp \left(-\bar{\mu}_{n^{*}} \tau\right) E\left[\exp \left(-\bar{\sigma}_{n} \bar{X} \sqrt{\tau}\right) 1_{Z \geq-\alpha}\right]
\end{array}
$$

one can use that

$$
(\bar{X}, Z) \sim \operatorname{Norm}_{2}\left(\left(\begin{array}{l}
0 \\
0
\end{array}\right),\left(\begin{array}{cc}
1 & \bar{\rho} \\
\bar{\rho} & 1
\end{array}\right)\right)
$$

where

$$
\bar{\rho}=\frac{\bar{\sigma}_{n}}{\sqrt{\sigma_{n}^{2}+\bar{\sigma}_{n^{*}}^{2}}}
$$

q.e.d.

Corollary 1. In the economy characterized by the equations (1)-(5) the price of an exchange rate call option has the following closed form representation:

$$
C_{t}=e_{t} \exp \left\{-R^{*}(T-t)\right\} \Pi_{1}-K \exp \{-R(T-t)\} \Pi_{2}
$$

where

$$
\begin{aligned}
& \Pi_{1}=\sum_{n} \sum_{n^{*}} p(n) p^{*}\left(n^{*}\right) \exp \left\{-\left(\bar{\mu}_{n^{*}}-\frac{\bar{\sigma}_{n^{*}}^{2}}{2}-R^{*}\right) \tau\right\} \Phi\left(d_{1, n, n^{*}}\right) \\
& \Pi_{2}=\sum_{n} \sum_{n^{*}} p(n) p^{*}\left(n^{*}\right) \exp \left\{-\left(\mu_{n}-\frac{\sigma_{n}^{2}}{2}-R\right) \tau\right\} \Phi\left(d_{2, n, n^{*}}\right)
\end{aligned}
$$

\section{CONCLUSION}

In this paper we developed a framework for asset pricing in the context of the general equilibrium model in Necula (2008). We analyzed a benchmark specification of this model consisting of constant growth rates and volatilities for the output and the money supply, and of log-normal amplitude of the jumps.

The dynamics equation of the nominal exchange rate depends on the real and nominal sectors of the two countries. The theorised distribution of the 
logarithm of the nominal exchange rate is a mixture of normal distributions, and is therefore leptokurtic.

We derived the fundamental evaluation PIDE for four different classes of assets. We also obtained a closed-form formula for pricing exchange rate call options in the context of this benchmark two-country economy.

\section{REFERENCES}

[1] Arditti, F., and K. John, (1980), "Spanning the State Space with Options," Journal of Financial and Quantitative Analysis 18, 1-9.

[2] Bailey, W., and R. Stulz, (1989), "The pricing of Stock Index Options in a General Equilibrium Model," Journal of Financial and Quantitative Analysis 24, 1-12.

[3] Bakshi, G., C. Cao, and Z. Chen, (1997), "Empirical Performance of Alternative Option Pricing Models," Journal of Finance 52, 2003-2049.

[4] Bakshi, G., and Z. Chen, (1997a), "An Alternative Valuation Model for Contingent Claims," Journal of Financial Economics 44, 123-165.

[5] Bakshi, G., and Z. Chen, (1997b), "Equilibrium Valuation of Foreign Exchange Claims," Journal of Finance 52, 799-826.

[6] Bakshi, G., and D. Madan, (2000), "Spanning and derivative-security valuation," Journal of Financial Economics 55, 205-238.

[7] Bakshi, G. S., and Z. Chen, (1996), "Inflation, Asset Prices, and the Term Structure of Interest Rates in Monetary Economies," The Review of Financial Studies 9, 241-275.

[8] Ball, C., and A. Roma, (1994), "Stochastic Volatility Option Pricing," Financial and Quantitative Analysis 29, 589-607.

[9] Bates, D., (1996), "Jumps and Stochastic Volatility: Exchange Rate Processes Implicit in Deutsche Mark Options," Review of Financial Studies 9, 69-107.

[10]Bick, A., (1987), "On the consistency of the Black-Scholes model with a general equilibrium framework," Journal of Financial and Quantitative Analysis 22, 259276.

[11]Bjork, T., (1998), Arbitrage Theory in Continuous Time, Oxford University Press.

[12]Black, F., and M. Scholes, (1973), "The pricing of options and corporate liabilities," Journal of Political Economy 81, 637-659.

[13] Breeden, D., (1979), "An Intertemporal Asset Pricing Model With Stochastic Consumption and Investment Opportunities," Journal of Financial Economics 7, 265-296.

[14]Breeden, D, (1986), "Consumption, Production, Inflation and Interest Rates: A Synthesis," Journal of Financial Economics 16, 3-40.

[15] Breeden, D., and R. Litzenberger, (1978), "Price of state contingent claims implicit in option prices," Journal of Business 51, 621-652.

[16] Cont, R., and P. Tankov, (2004), Financial modeling with jump processes, CRC Press.

[17] Cox, J. C., and C. Huang, (1989), "Optional consumption and portfolio policies when assets prices follow a diffusion process," Journal of Economic Theory 49, 33-83. 
[18] Cox, J. C., J. E. Ingersoll, and S. A. Ross, (1985a), "An Intertemporal General Equilibrium Model of Asset Prices," Econometrica 53, 363-384.

[19] Cox, J. C., J. E. Ingersoll, and S. A. Ross, (1985b), "A Theory of the Term Structure of Interest Rates," Econometrica 53, 385-407.

[20] Detemple, J., and L. Selden, (1991), "A general equilibrium analysis of option and stock market interactions," International Economic Review 32, 279 -303.

[21] Duffie, D., J. Pan, and K. Singleton, (2000), "Transform Analysis and Option Pricing for Affine Jump-Diffusions," Econometrica 68, 1343-1376.

[22]Fleming, W., and R. Rishel, (1975), Deterministic and Stochastic Optimal Control, Springer Verlag.

[23] Heston, S., (1993), "A Closed-Form Solution for Options with Stochastic Volatility with Applications to Bond and Currency Options," Review of Financial Studies 6, 327-343.

[24] Hull, J., (1993), Options, Futures, and other Derivative Securities, Prentice Hall, Englewood Cliffs.

[25]Hull, J., and A. White, (1987), "The Pricing of Options with Stochastic Volatilities," Journal of Finance 42, 281-300.

[26] Jacod, J., and A. N. Shiryaev, (2003), Limit Theorems For Stochastic Processes, 2nd Edition, Springer Verlag.

[27] Kou, S. G., (2002), "A jump diffusion model for option pricing," Management Scence. 48, 1086-1101.

[28]Lucas, R., (1978), "Asset Prices in an Exchange Economy," Econometrica 46, 1429-1445.

[29] Lucas, R., (1982), "Interest Rates and Currency Prices in a Two-Country World," Econometrica 10, 335-360.

[30] Merton, R. C., (1973a), "An Intertemporal Capital Asset Pricing Model," Econometrica 41, 867-887.

[31] Merton, R. C., (1973b), "Theory of Rational Option Pricing," Bell Journal of Economics and Management Science 4, 141-183.

[32] Merton, R. C., (1976), "Option Pricing When Underlying Stock Returns Are Discontinuous," Journal of Financial Economics 3, 125-144.

[33] Naik, V., and M. Lee, (1990), "General Equilibrium Pricing of Options on the Market Portfolio with Discontinuous Returns," The Review of Financal Studies 3, 493-521.

[34] Necula, C, (2008), “A Two-Country Discontinuous General Equilibrium Model”, http://papers.ssrn.com/sol3/papers.cfm?abstract id=1289413

[35] Oksendal, B., (2000), Stochastic Differential Equations, 5th Edition, Springer Verlag.

[36] Protter, P., (1992), Stochastic Integration and Differential Equations, Springer Verlag.

[37] Revuz, D., and M. Yor, (1991), Continuous Martingales and Brownian Motion, Springer Verlag.

[38] Samuelson, P., (1965), "Rational Theory of Warrant Pricing," Industrial Management Review 6, 13-32.

[39] Scott, L. O., (1997), "Pricing Stock Options in a Jump-diffusion Model with Stochastic Volatility and Interest Rates: Application of Fourier Inversion Methods," Mathematical Finance 7, 345-358. 
[40] Singleton, K. J., (2001), "Estimation of Affine Asset Pricing Models Using the Empirical Characteristic Function," Journal of Econometrics 102, 111-141.

[41] Stapleton, R. C., and M. G. Subrahmanyam, (1978), "A Multiperiod Equilibrium Asset Pricing Model," Econometrica 46, 1077-1096.

[42] Stein, E., and J. Stein, (1991), "Stock Price Distributions with Stochastic Volatility: an Analytic Approach," Review of Financial Studies 4, 727-752.

[43] Stulz, R. M., (1987), "An Equilibrium Model of Exchange Rate Determination and Asset Pricing with Nontraded Goods and Imperfect Information," The Journal of Political Economy 95, 1024-1040.

[44] Vasicek, O., (1977), "An Equilibrium Characterization of the Term Structure," Journal of Financial Economics 5, 177-188. 